\title{
Metastatic Adenocarcinoma to the Wrist Presenting as Carpal Tunnel Syndrome: A Report of an Unusual Cause
}

\author{
SA Roohi, MS Orth, OC Samsudin*, MS Orth, MH Shukur**, MS Orth, S Ibrahim**, FRCS \\ Department of Orthopaedic, Universiti Putra Malaysia, Serdang, Malaysia, \\ ${ }^{*}$ Gleneagles Intan Medical Centre, Kuala Lumpur, Malaysia. \\ ${ }^{* *}$ Department of Orthopaedics and Traumatology, Universiti Kebangsaan Malaysia, Kuala Lumpur, Malaysia
}

\section{INTRODUCTION}

Secondary metastases to the hand are rare, accounting for $0.1 \%$ of all metastases, and commonly involve bones ${ }^{1,2}$. Soft tissue involvement is extremely unusual although it is significantly more common in carcinoma than sarcoma. Metastatic lesions of adenocarcinoma of the rectum to the liver, lung and vertebrae are well known. Here we report an unusual site of metastatic deposit of primary adenocarcinoma of the rectum to the soft tissues of the wrist causing carpal tunnel syndrome, which if correctly diagnosed earlier from the symptoms could have prevented an untimely amputation.

\section{CASE REPORT}

A 57 year-old female was first seen in the orthopaedic clinic for right hand pain and swelling over the volar aspect of the wrist of five months duration. The swelling had been progressively enlarging and was associated with increasing pain, tingling and numbness involving the radial three digits. The pain was not severe but refractory to nonsteroidal antiinflammatory drugs. Her right hand was noted to have appreciable weakness and stiffness.

Five years ago, the patient was diagnosed with carcinoma of the rectum and underwent a low anterior resection 3 months after the initial diagnosis. There was a recurrence twenty months after the first surgery at which time a resection and permanent colostomy was performed. Following courses of adjuvant chemo- and radiotherapy she was well until almost two years later the development of a persistent cough prompted a chest radiograph which revealed multiple lung metastases. One year later ( 5 months prior to being seen in the orthopaedic clinic), she noted a swelling in her right hand, but a radiograph at the time did not show any bony involvement. Subsequent pain and progressive swelling prompted an orthopaedic referral and further investigation.

On examination, the entire right hand appeared swollen particularly over the midpalmar and ulnar aspects, but with no apparent wasting of the small muscles of the hand. There was a poorly defined lump of approximately $5 \times 6 \mathrm{~cm}$ centred over the palmar aspect (Figure 1). The mass was firm to hard and non-pulsatile and the overlying skin was shiny and stretched. There was appreciable anhydrosis of the radial aspect of the hand and sensation was reduced in the radial three digits. The abductor pollicis brevis was grade 3 on the MRC scale. The Tinel percussion test of the median nerve was positive.

Plain radiograph of the affected hand showed an ulnar-sided soft tissue shadow with a sclerotic hamate bone (Figure 2) and a lytic lesion at the base of the fifth metacarpal. MRI revealed tumour involvement of most of the bones of the hand except the scaphoid, trapezium, thumb phalanges and second metacarpal. Bony involvement seen in both T1- and T2-weighted images was of intermediate intensity. The tumour pushed the flexor tendons radially and tumour extension into the carpal tunnel could be seen in early axial cuts. Guyon's canal was spared.

A month later, a decision was made to decompress the carpal tunnel as a palliative measure. Under regional brachial plexus block, carpal tunnel release using a flexor carpi radialis approach and fine needle aspiration of the tumour was performed. There was short-lived pain relief and improvement in the level of numbness.

This operative findings were predictive of two factors: it would only be a matter of time before all symptoms recurred and that the pain was not only from the tumour compression, but also highly likely from the tumour involvement of multiple bones. Thus simple excision of the tumour would not only have been challenging but also ineffective. Cytological results confirmed a metastatic adenocarcinomatous lesion. Due to the increasing severity of local pain that was refractory to medication, and in view of the extent of the tumour (bony and soft tissue infiltration), a below-elbow amputation was performed 3 months following the primary release and biopsy. Dissection of the gross specimen revealed a dumbbell-shaped tumour mass compressing the median nerve (Figure 3). Histopathologic examination of the amputated specimen disclosed well 


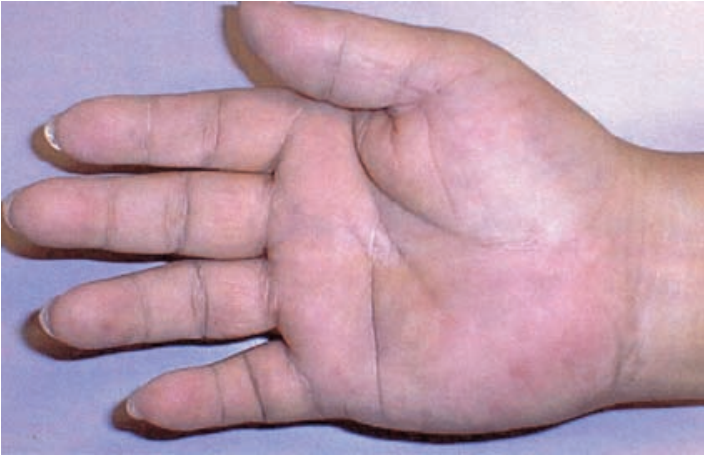

Fig. 1: Hypothenar/midpalmar swelling with appreciable dryness of the radial three digits and radial half of the palm

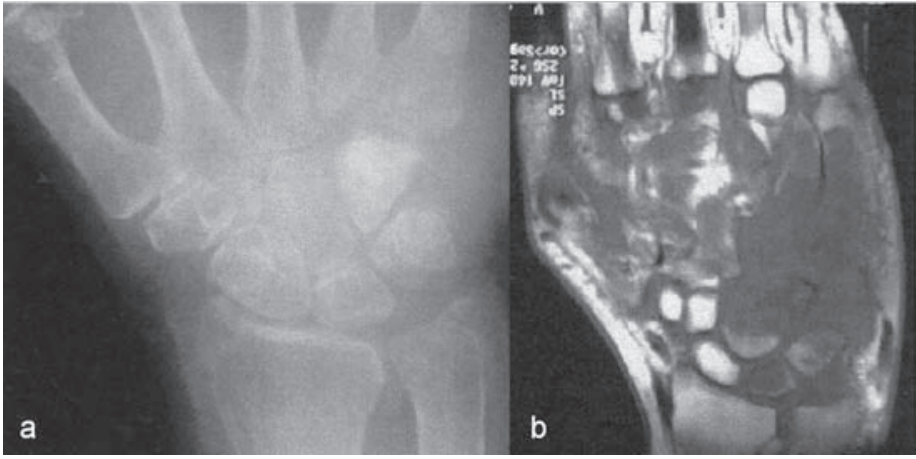

Fig. 2: Plain radiograph (a) shows ulnar sided soft tissue shadow and a sclerotic hamate bone. MRI (b) revealing areas of bony involvement.

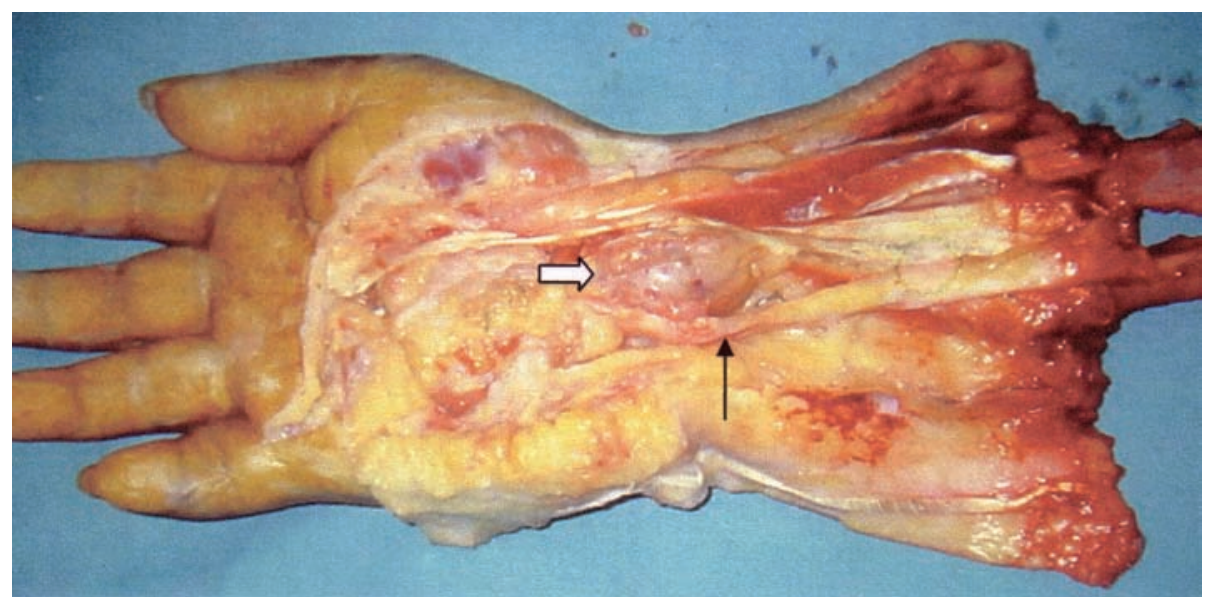

Fig. 3: Gross specimen dissection showing a dumbbell-shaped tumor (white arrow) compressing on the median nerve (black arrow).

differentiated malignant cells in a glandular pattern infiltrating the muscle. The median and ulnar nerve bundles were not infiltrated by malignant cells. On last follow-up, eight months post-operatively, the amputation stump was well healed, but the patient had phantom pain and was referred to the pain clinic.

\section{DISCUSSION}

Adenocarcinoma of the rectum is well known to metastasize, especially to the liver, lung and spine. In general, metastases to the hand are rare $^{1-3}$. The bones of the hand are predominantly affected with the terminal phalanges being the most commonly involved followed by the proximal phalanges and the metacarpals ${ }^{2}$. The preponderance for metastatic involvement of bones of the hand is not completely understood but hematogenous spread and the paucity of red marrow in the phalanges and metacarpals has been thought to be the reason for this infrequent occurrence ${ }^{3}$. Soft tissue involvement is even rarer representing approximately $10 \%$ of all hand metastases with $25 \%$ having both bony and soft tissue involvement ${ }^{2}$. Even though our patient had involvement of both bones and soft tissue, we believe the soft tissue involvement was earlier and therefore the cause was missed at initial presentation.

The proximity of the soft tissue involvement provides explanation for the presenting problem in this case. The list of causes of carpal tunnel syndrome is extensive but space occupying lesions of the carpal tunnel are often categorized as benign or primary malignant lesions. There are several reports on malignant lesions as the underlying cause of carpal tunnel syndrome $e^{4.5}$. However all these causes are mainly malignant tumours arising primarily from the tissues in the vicinity of the carpal tunnel. In our case, the lesion that caused carpal tunnel syndrome was a metastatic deposit. Thus, this is a rare and unusual cause of carpal tunnel syndrome. The treatment of such cases remains palliative, but if detected early enough may lead to a longer morbidity free interval and avoid or at least delay ablative surgery. 
Metastatic Adenocarcinoma to the Wrist Presenting as Carpal Tunnel Syndrome: A Report of an Unusual Cause

\section{REFERENCES}

1. Amadio PC, Lombardi RM. Metastatic tumors of the hand. J Hand Surg 1987; 12A: 311-6.

2. Kerin R. Metastatic tumors of the hand. J Bone Joint Surg 1983; 65A(9): 1331-5.

3. Kerin R. The hand in metastatic disease. J Hand Surg 1987; 12A (1): 77-83.

4. Nather A, Chong PY. A rare cause of carpal tunnel syndrome due to tenosynovial osteochondroma. J Hand Surg 1986; 11B: 47880.

5. Wood MK, Erdmann MW, Davies DM. Malignant schwannoma mistakenly diagnosed as carpal tunnel syndrome. $J$ Hand Surg 1993; $18 B(2): 187-8$. 